\title{
A protective Zika virus E-dimer-based subunit vaccine engineered to abrogate antibody- dependent enhancement of dengue infection
}

\author{
Jose Luis Slon-Campos ${ }^{1,12}$, Wanwisa Dejnirattisai ${ }^{1,12}$, Brett W. Jagger 2,11, César López-Camacho ${ }^{\mathbb{N}^{3}}$, \\ Wiyada Wongwiwat ${ }^{1}$, Lorellin A. Durnell2 ${ }^{2}$, Emma S. Winkler ${ }^{2}$, Rita E. Chen ${ }^{2}$, Arturo Reyes-Sandoval $\mathbb{1}^{3}$, \\ Felix A. Rey ${ }^{4,5}$, Michael S. Diamond (1) ${ }^{2,6,7,8}$, Juthathip Mongkolsapaya ${ }^{1,9 \star}$ and Gavin R. Screaton (1) ${ }^{10 \star}$
}

\begin{abstract}
Infections with dengue virus (DENV) and Zika virus (ZIKV) can induce cross-reactive antibody responses. Two immunodominant epitopes-one to precursor membrane protein and one to the fusion loop epitope on envelope (E) protein-are recognized by cross-reactive antibodies ${ }^{1-3}$ that are not only poorly neutralizing, but can also promote increased viral replication and disease severity via Fc $\gamma$ receptor-mediated infection of myeloid cells-a process termed antibody-dependent enhancement (ADE) ${ }^{1,4,5}$. ADE is a significant concern for both ZIKV and DENV vaccines as the induction of poorly neutralizing cross-reactive antibodies may prime an individual for ADE on natural infection. In this report, we describe the design and production of covalently stabilized ZIKV E dimers, which lack precursor membrane protein and do not expose the immunodominant fusion loop epitope. Immunization of mice with ZIKV E dimers induces dimer-specific antibodies, which protect against ZIKV challenge during pregnancy. Importantly, the ZIKV E-dimer-induced response does not cross-react with DENV or induce ADE of DENV infection.
\end{abstract}

Following the Pacific Islands epidemics in 2013 and 2014, Zika virus (ZIKV) spread across South America, leading to an explosive outbreak in Brazil in 2015. Although most ZIKV infections lead to asymptomatic or mild disease, severe neurological sequelae can ensue, including Guillain-Barré syndrome (which was first recognized after the French Polynesian outbreak ${ }^{7}$ ), and microcephaly and other congenital anomalies (which were observed in children born to mothers who suffered a ZIKV infection during pregnancy ${ }^{8}$ ). There has been much study of ZIKV since the outbreak in Brazil, which has clarified several key features of ZIKV pathogenesis, including the ability of the virus to infect and cross the placenta, targeting specific neuroprogenitor cells in the developing fetal brain'. Because of the initial high infection rate in a naive population in South America, the ZIKV outbreak created herd immunity, which has essentially terminated the epidemic, although sporadic cases still occur. However, the catastrophic consequences of infec- tion during pregnancy mandate the development of ZIKV vaccines, for as herd immunity wanes, the likelihood of another epidemic will increase. Several ZIKV vaccine platforms are currently in development, including inactivated viruses, live attenuated viruses, viralvectored vaccines, and protein subunit- and nucleic acid-based formulations expressing envelope (E) proteins. These have shown protection in small and large animal models of ZIKV infection, with several in early-phase human clinical trials ${ }^{10}$.

The ability to generate large numbers of human monoclonal antibodies (mAbs) from flavivirus-infected patients has expanded our knowledge of the breadth and specificity of the human response to infection, and allowed the delineation of antigenic sites that produce poorly or potently neutralizing antibody responses. The fusion loop epitope (FLE) is an immunodominant epitope on the E protein, and antibodies to this highly conserved region frequently show broad flavivirus cross-reactivity ${ }^{2-4,11}$. FLE-reactive antibodies generated from dengue virus (DENV)- or ZIKV-infected patients show low or absent neutralization of ZIKV, yet can promote antibody-dependent enhancement (ADE) of ZIKV infection ${ }^{12,13}$. Whether pre-existing DENV immunity can prime ADE to ZIKV remains controversial; in murine models, DENV antibodies can enhance ZIKV infection and vice versa ${ }^{14-16}$, whereas small-scale primate studies have not reproduced this ${ }^{17-19}$. Clinical studies suggest that previous DENV immunity has no detrimental effect on ZIKV infection but may protect them from symptomatic disease ${ }^{20,21}$.

Some of the best neutralizing mAbs to DENV and ZIKV recognize conformational epitopes, such as E domain III or quaternary epitopes containing two or more copies of $\mathrm{E}^{14,22-24}$. Given their diversity, antibodies recognizing epitopes beyond the monomeric conformation of Es can show narrow restriction to ZIKV or a single serotype of DENV, or can be broadly reactive. A recently described class of human mAbs recognize the E-dimer epitope (EDE) - a quaternary epitope formed across the interface of two E monomers arranged in a head-to-tail conformation ${ }^{25}$. The highly conserved nature of the EDE allows some EDE mAbs to neutralize all four

'Wellcome Centre for Human Genetics, Nuffield Department of Medicine, University of Oxford, Oxford, UK. ²Department of Medicine, Washington University School of Medicine, Saint Louis, MO, USA. ${ }^{3}$ Jenner Institute, Nuffield Department of Medicine, University of Oxford, Oxford, UK. ${ }^{4}$ Unité de Virologie Structurale, Département de Virologie, Institut Pasteur, Paris, France. ${ }^{5}$ Centre National de la Recherche Scientifique, Unité Mixte de Recherche 3569, Paris, France. ${ }^{6}$ Department of Pathology and Immunology, Washington University School of Medicine, Saint Louis, MO, USA. ${ }^{7}$ Department of Molecular Microbiology, Washington University School of Medicine, Saint Louis, MO, USA. ${ }^{8}$ Andrew M. and Jane M. Bursky Center for Human Immunology and Immunotherapy Programs, Washington University School of Medicine, Saint Louis, MO, USA. 'Dengue Hemorrhagic Fever Research Unit, Office for Research and Development, Faculty of Medicine, Siriraj Hospital, Mahidol University, Bangkok, Thailand. ${ }^{10}$ Division of Medical Sciences, University of Oxford, Oxford, UK. "Present address: Department of Medicine, Western Michigan University Homer Stryker MD School of Medicine, Kalamazoo, MI, USA. ${ }^{12}$ These authors contributed equally: Jose Luis Slon-Campos, Wanwisa Dejnirattisai. *e-mail: jmongkol@well.ox.ac.uk; gavin. screaton@medsci.ox.ac.uk 


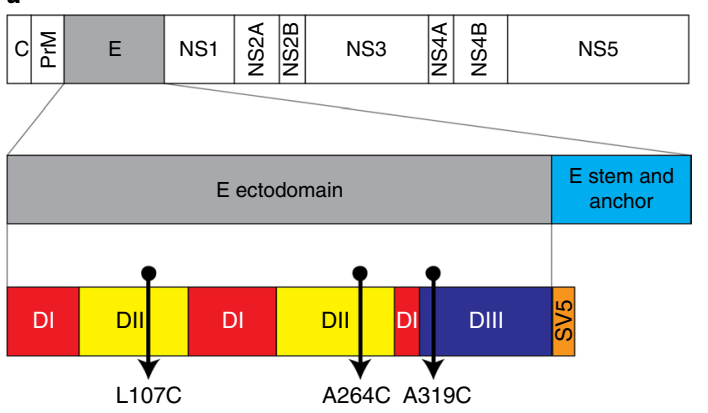

c
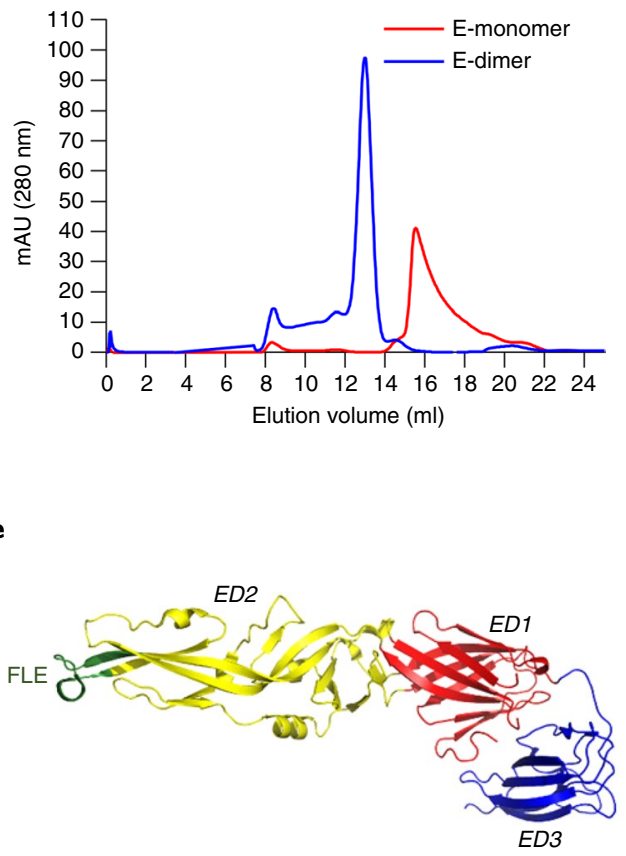

$\mathbf{f}$

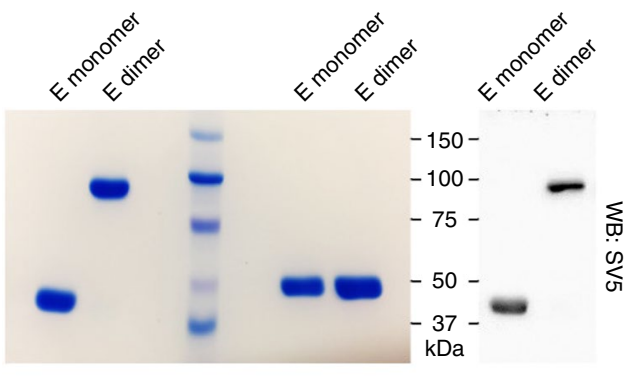

Non-reducing Reducing Non-reducing

d
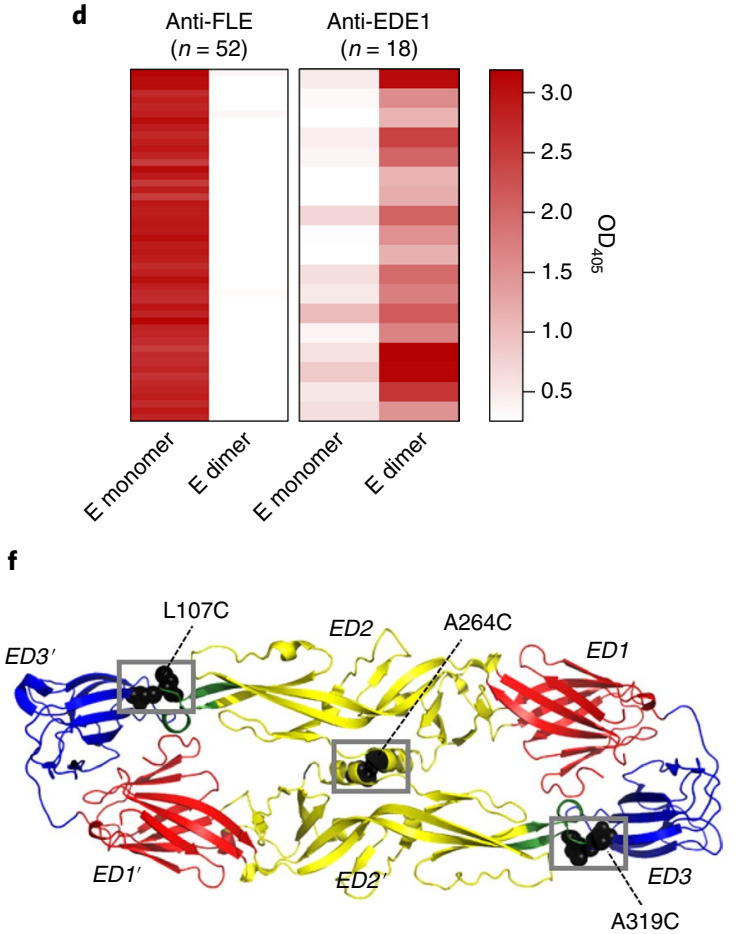

Fig. 1 | Engineering, production and assessment of ZIKV E-monomer and E-dimer proteins. a, Schematic of the ZIKV E protein within the context of the viral polyprotein. The localization of the residues that form interchain disulfide bonds on mutation to cysteine is noted. D, domain; C, capsid protein; prM, precursor membrane protein; NS, non-structural protein; SV5, Simian virus 5-derived tag (sequence GKPIPNPLLGLD). b, Coomasie-stained SDS-PAGE and immunoblotting (WB) analysis of the ZIKV E-monomer and E-dimer proteins under non-reducing and reducing (10\% $\beta$-ME) conditions. c, Superimposed SEC elution profiles of purified ZIKV E-monomer and E-dimer proteins, showing the different peak elution volumes for each construct. $\mathrm{mAU}$, milliabsorbance units. $\mathbf{b}$ and $\mathbf{c}$ are representative of the protein batches used throughout the study ( $n=3$ and 2 for the ZIKV E-monomer and E-dimer protein batches, respectively; two independent experiments were performed for each batch, and all of the results were reproducible). $\mathbf{d}$, Heat maps showing the intensity of binding of 52 anti-FLE and 18 anti-EDE1 human mAbs isolated from DENV-infected patients to purified ZIKV E-monomer and E-dimer proteins in ELISA. Data are presented as mean readings of optical density measured at a wavelength of $405 \mathrm{~nm}\left(\mathrm{OD}_{405}\right)$, which were obtained for all of the protein batches used in the study ( $n=3$ and 2 protein preparations for ZIKV E monomer and E dimer, respectively). e,f, Monomeric (e) and dimeric structures (f) of the ZIKV E protein (generated from the Protein Data Bank reference 5jhm using PyMOL software), indicating the ED1 (red), ED2 (yellow) and ED3 domains (blue), as well as the FLE (green). For the covalent E dimer, the positions of the cysteine-mutated residues (black) and the resulting interchain disulfide bonds (gray boxes) are indicated.

DENV serotypes and ZIKV ${ }^{13,26,27}$, whereas most other mAbs targeting quaternary epitopes are highly specific. The mAbs HM14c10, 5J7 and ZIKV-117 bind to epitopes defined by the arrangement of two or more E dimers on the viral surface of DENV1, DENV3 and ZIKV, respectively ${ }^{28-30}$, while the epitopes targeted by the mAbs 2D22 and ZIKV-195 are contained within the E-dimer interfaces of DENV2 and ZIKV ${ }^{31,32}$. Furthermore, antibodies to complex quaternary epitopes such as the EDE are resistant to ZIKV escape mutation $^{26}$, whereas virus escape from E domain III-specific mAbs can lead to the rapid generation of viral escape mutants ${ }^{33,34}$.

As both precursor membrane protein (prM) and the FLE induce the formation of poorly neutralizing yet enhancing antibodies ${ }^{1-3,22}$, we sought to develop a subunit ZIKV immunogen that would not induce these responses. We previously described the production of stable DENV dimers, formed by the introduction of one or two interchain disulfide bonds $\mathrm{s}^{35,36}$. The introduction of a single disulfide bond does not reduce exposure of the FLE, whereas the introduction of two disulfide bonds reduces the binding of some anti-FLE mAbs, but does not completely abolish FLE recognition. For ZIKV, we chose to combine these two approaches to produce $\mathrm{E}$ dimers stabilized by the introduction of three mutations in ZIKV E (L107C, A264C and A319C) (Fig. 1a). Recombinant monomeric or dimeric ZIKV E was produced by HEK293T cell expression and purified by sequential affinity and size exclusion chromatography (SEC) (Fig. 1b,c). 


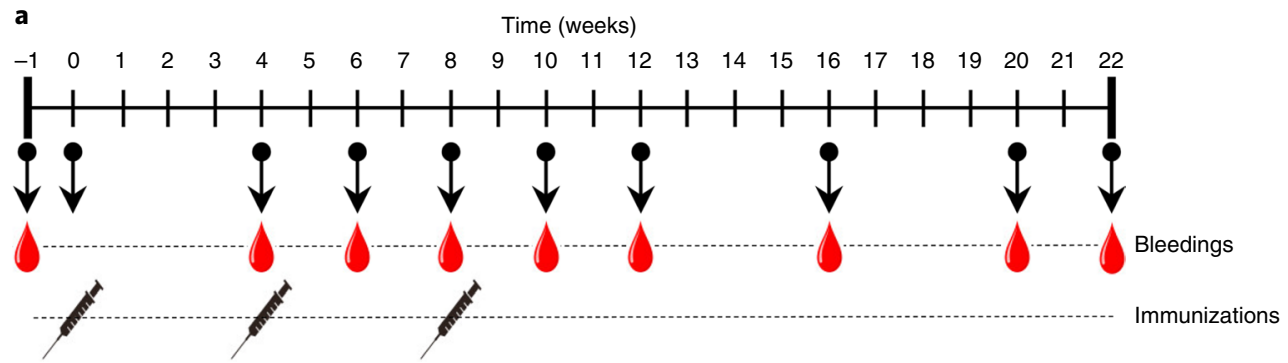

b

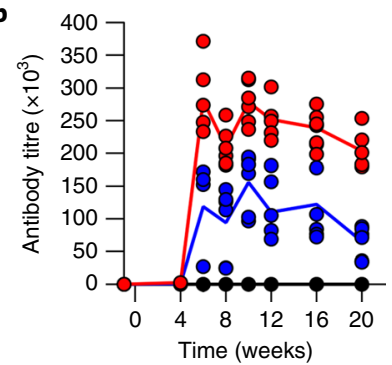

d

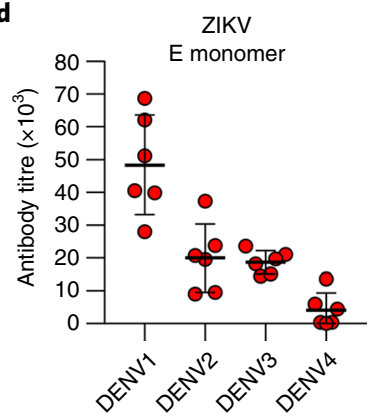

c
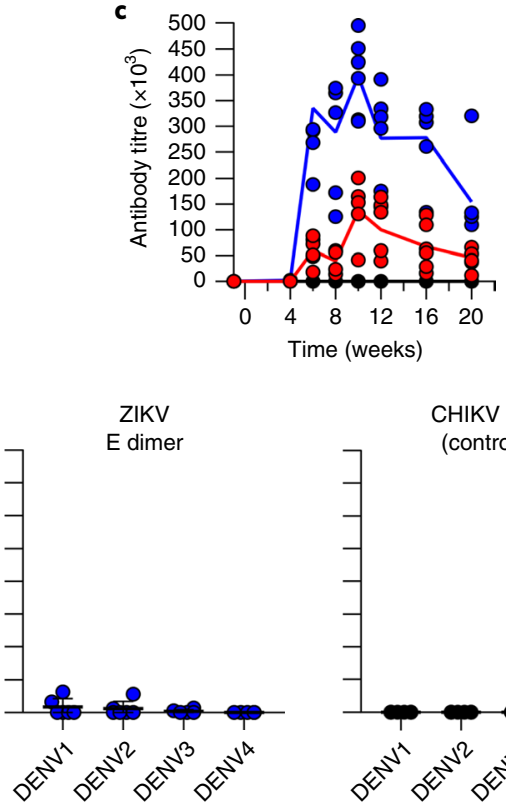

- E- E monomer

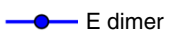

$\longrightarrow$ CHIKV E2

Fig. 2 | Binding properties of mice sera following immunization with ZIKV E-monomer and E-dimer proteins. a, Schematic of the immunization protocol. Wild-type BALB/C mice ( $n=6$ animals per group) were immunized with $10 \mu \mathrm{g}$ E-monomer, E-dimer or CHIKV E2 protein, and boosted twice at 4-week intervals, with serum collected as shown. b,c, ELISA analyses showing the antibody titers obtained from immunized-mouse serum on captured ZIKV PF13 virions (b) and ZIKV E dimers (c) ( $n=6$ individual mouse serum samples per group for each time point; individual data points and arithmetic means (solid lines) are shown). d, DENV cross-binding of ZIKV E-monomer (left), ZIKV E-dimer (middle) and CHIVK E2 (right) immune sera, determined as in b using serum samples obtained 10 weeks after priming. Individual data points are shown, along with arithmetic means \pm s.d. (for each virus, $n=6$ individual mouse serum samples per group).

The introduction of three disulfide bridges recapitulates the EDE, as shown by the binding of many EDE1 mAbs to the E-dimer but not to the E-monomer protein (Fig. 1d). EDE1 antibodies differ from EDE2 antibodies, as EDE2 antibodies require glycan at position N153 of the E protein for optimal binding. EDE1 and EDE2 both bind to and neutralize DENV, whereas only EDE1 shows potent neutralizing activity against $\mathrm{ZIKV}^{13,25}$. In addition, and in contrast with the single and double mutants mentioned above, the triple-disulfide-bonded E dimer is completely locked such that it does not expose the FLE or bind to any of a panel of 52 FLE-reactive mAbs generated from DENV-infected patients (Fig. 1d). Molecular representations of monomeric wild-type ZIKV $\mathrm{E}$ and the engineered dimeric ZIKV E mutant are also shown (Fig. 1e,f).

We immunized BALB/c mice at 0,4 and 8 weeks with $10 \mu \mathrm{g}$ of either ZIKV E monomer or ZIKV E dimer suspended in the adjuvant AddaVax, and compared the responses versus immunization with a chikungunya virus E2 protein (CHIKV E2) control. The immunization and bleeding schedule is shown in Fig. 2a. Serum from vaccinated mice was tested for reactivity to ZIKV virions or recombinant $\mathrm{E}$ dimers by enzyme-linked immunosorbent assay (ELISA). Reactivity to whole ZIKV virions was slightly higher in mice immunized with ZIKV E monomer versus E dimer (Fig. 2b), whereas reactivity to recombinant $\mathrm{E}$ dimers was clearly higher in the mice immunized with E dimers (Fig. 2c). Next, we evaluated the cross-reactivity of serum from the two groups of ZIKV-immunized mice to the four DENV serotypes by ELISA. E-monomerimmunized mice cross-reacted to DENV, which was much reduced or absent in the serum from E-dimer-immunized mice-probably due to the hidden FLE, and consequent failure to generate an antiFLE response by the ZIKV E dimer (Fig. 2d).

Neutralization assays against ZIKV and the four DENV serotypes were performed using a focus reduction neutralization test (FRNT). As expected, serum-neutralizing activity against ZIKV was undetectable in animals immunized with CHIKV E2. In comparison, neutralizing activity $\left(\mathrm{FRNT}_{50}\right.$ titers-dilution of serum to obtain a $50 \%$ reduction in the number of foci-of 496.4 and 535.8, and $\mathrm{FRNT}_{90}$ titers of 96.7 and 148.4, for $\mathrm{E}$ monomer and $\mathrm{E}$ dimer, respectively) was observed in the E-dimer- and E-monomervaccinated mice, with higher levels seen in the E-dimer group (Fig. 3a). As detailed below, similar results were also obtained when immunizing different groups and strains of mice (Supplementary Figs. 1 and 3). Notably, the serum from mice immunized with the ZIKV E monomer showed some cross-neutralization of DENV, whereas little (if any) cross-neutralization of DENV was seen in the ZIKV E-dimer-immune serum (similar to the CHIKV E2 control) (Fig. 3b). 


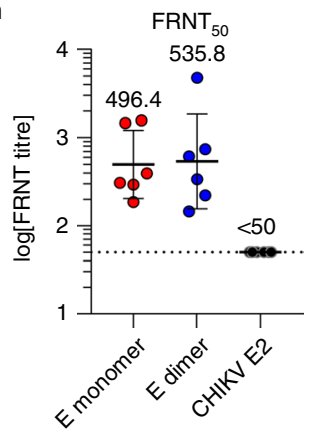

b

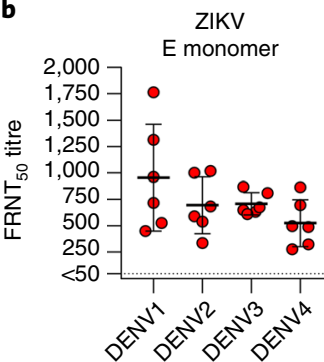

c

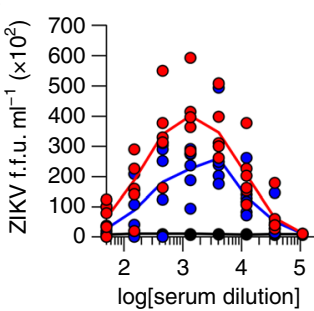

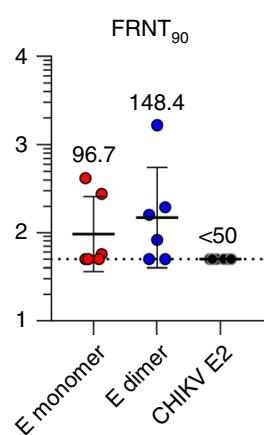

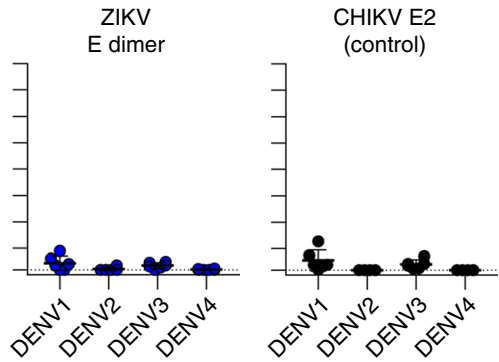

d

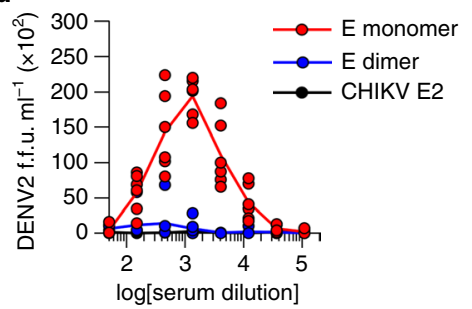

Fig. 3 | Neutralizing and ADE activities of mouse sera following immunization with the ZIKV E-monomer and E-dimer proteins. Serum was collected from immunized female BALB/c mice at 10 weeks post-prime and analysed for ZIKV and DENV neutralization activity by FRNT assay. a, FRNT $_{50}$ (left) and FRNT $_{90}$ values (right) against ZIKV were calculated for individual animals in each group. The dashed lines indicate the limit of detection of the assay. Individual measurements, as well as geometric mean \pm s.d. values, are noted (in both cases, $n=6$ individual mouse serum samples per group). b, FRNT $_{50}$ titers against the four DENV serotypes were calculated for animals immunized with ZIKV E monomer (left), ZIKV E dimer (middle) and CHIVK E2 (right). Individual measurements, as well as arithmetic mean \pm s.d. values, are shown (for each virus, $n=6$ individual mouse serum samples per group). c,d, Infection enhancement curves of U937 cells infected with ZIKV PF13 (c) and DENV2 16681 viruses (d) in the presence of serially diluted mouse immune serum (for each virus, $n=6$ individual mouse serum samples per group). Data are shown as single data points and arithmetic means (solid lines).

Almost all mAbs against flaviviruses generated to date will induce $\mathrm{ADE}$ against the homologous virus strain when diluted to subneutralizing concentrations ${ }^{37}$. To evaluate the effects of the different immunogens on ADE, we tested the ability of ZIKV immune serum to induce ADE of ZIKV or DENV2. As expected, both ZIKV E-monomer and E-dimer immunization schedules induced antibodies capable of enhancing ZIKV infection, although peak enhancement titers were lower in E-dimer- compared with E-monomer-immunized mice (Fig. 3c). However, and in agreement with the markedly reduced binding of serum to DENVs, ADE of DENV2 was almost absent in the E-dimer-immune serum (Fig. 3d). Consistently, immunization of outbred CD1 mice under the same conditions resulted in antibody responses with similar binding, neutralizing and enhancing activities (Supplementary Fig. 1).

To gain more insight into the polyclonal antibody responses to the E-monomer and E-dimer immunogens, we developed a series of yeasts displaying ZIKV E protein on the cell surface. Saccharomyces cerevisiae expressing all three E domains (ED123 (full-length E), domains I and II (ED12), and domain III only (ED3)) were constructed, which allowed us to map, in broad terms, where on the ZIKV E-protein polyclonal serum antibodies bind. It should be noted that the expression of the different proteins on the surface of yeast differs in the order ED3 > ED12>ED123, so that the absolute level of binding does not correlate directly with the titer of antibody in the serum. Another feature of this expression system is that $\mathrm{E}$ is inserted into the yeast surface in such a way that it is unable to form dimers, and thus only presents monomeric $\mathrm{E}$, which does not bind to E-dimer-specific antibodies. Figure 4a shows that, contrary to FLE- and ED3-restricted mAbs, EDE dimer-specific mAbs do not bind to yeasts displaying ZIKV-ED123.

The reactivity of serum from immunized mice to ED123, ED12 and ED3 was determined by flow cytometry. Both immunogens induced robust ED3 responses (Fig. 4b), although titers (halfmaximum binding) were reduced around fourfold when dimers and monomers were compared, which may be a consequence of the reduced exposure of the ED3 epitopes on the locked E dimer. Relative to E-monomer-immunized mice, the E-dimer-immune serum reactivity to ED12 was reduced around tenfold (Fig. 4c), whereas reactivity to ED123 was reduced around twofold (Fig. 4d).

To determine whether the reduced ED12 reactivity of E-dimerversus E-monomer-immune sera was due to the lack of induction of the immunodominant FLE response in the E-dimer-immunized mice, we constructed a yeast expressing ED12 in which tryptophan 101 is mutated to alanine (W101A). This destroys the FLE, preventing the binding of FLE-reactive antibodies ${ }^{38}$ (Fig. 4e). ED12 W101A binding (solid lines) was markedly reduced compared with unmutated ED12 (dashed lines) when incubated with serum from the E-monomer-immunized mice (around a fivefold reduction), whereas the binding of serum from the E-dimer-immunized mice was much less affected ( 0.2-fold reduction) (Fig. $4 \mathrm{e})$. In agreement with previous data, binding to yeast expressing DENV2 ED12 showed less cross-reactivity of the dimer- versus monomer-immune serum to DENV (around a fivefold difference) (Fig. 4f). This shows that the locked E dimers suppress the generation of an anti-FLE response, which accounts for most of the ED12 reactivity differences observed between E-monomer- and E-dimer-immune sera.

To further examine the neutralization characteristics of the polyclonal serum, we sought to deplete specific components of the response from serum before performing neutralization assays ${ }^{39}$. However, E monomer cannot be used to deplete serum antibodies in isolation because, in solution, E-dimer-specific antibodies can assemble $\mathrm{E}$ monomers into $\mathrm{E}$ dimers, meaning that $\mathrm{E}$ monomers will deplete both monomer- and dimer-specific antibodies ${ }^{35}$. To overcome this limitation, we used the yeast described above, displaying monomeric E protein for depletions, which do not bind or deplete quaternary E-dimer-specific antibodies.

ZIKV immune serum was incubated with yeast cells expressing a series of ZIKV E-derived antigens, which were then removed by centrifugation. The serum was checked for complete depletion by flow cytometry of ZIKV-expressing yeast particles (Supplementary Fig. 2). In the case of E-dimer depletion, the serum was first depleted of E-monomer-reactive antibodies using ED123 yeast, and then the E-dimer-reactive fraction was removed using sepharose beads coated with ZIKV E-dimer proteins. Depletion of E-dimer reactivity was confirmed by ELISA. We then tested the depleted serum for neutralization of ZIKV (Fig. 4g).

Depletion of E-monomer-immune serum with yeast expressing ED123 removes almost all of the neutralizing activity. Depletion with ED12 likewise removes the majority of the neutralizing activity, whereas depletion with ED3 leaves most of the neutralizing 

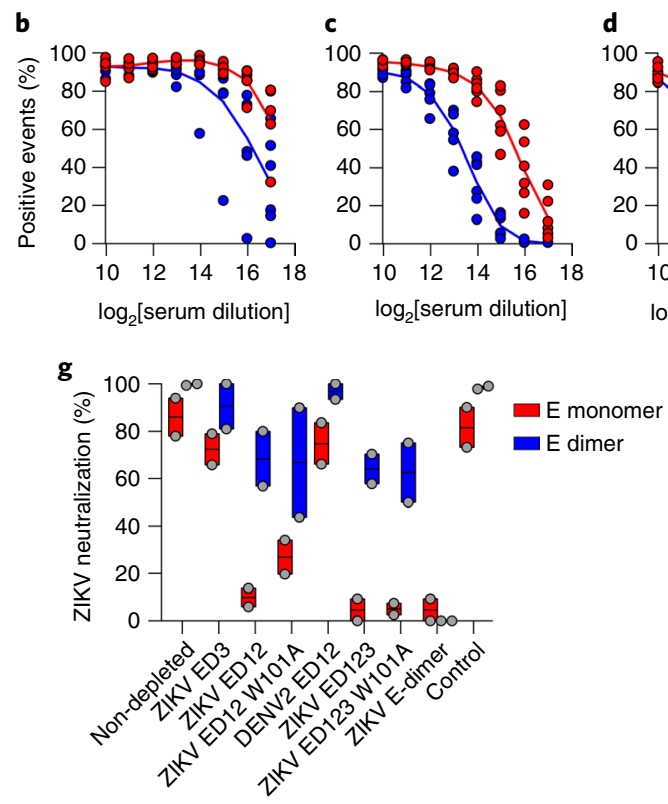
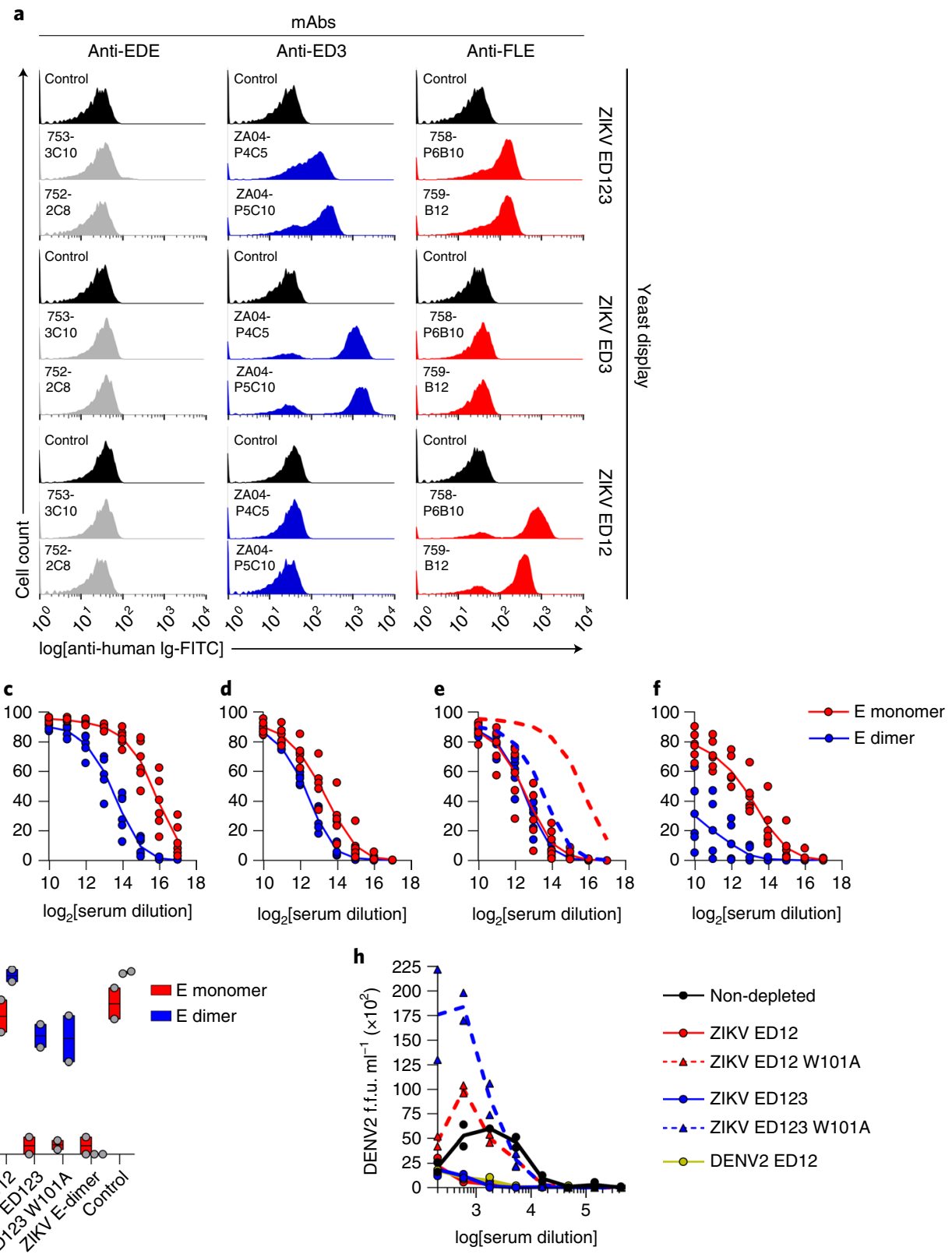

Fig. 4 | In-depth characterization of the immune response to ZIKV E monomers and E dimers. a, Flow cytometry profiles of ZIKV ED123-displaying yeast incubated with human anti-EDE (left), anti-ED3 (middle) and anti-FLE mAbs (right). Profiles of yeast in the absence of primary antibody were used as controls. The data shown are representative of three independent experiments. b-f, Flow cytometry profiles of E-monomer and E-dimer mouse immune serum against yeast displaying ZIKV ED3 (b; $4.61 \times 10^{5}$ and $1.22 \times 10^{5}$ titers for E monomer and E dimer, respectively), ED12 (c; $4.93 \times 10^{4}$ and $5.60 \times 10^{3}$ titers for E monomer and E dimer, respectively), ED123 (d; $8.56 \times 10^{3}$ and $4.24 \times 10^{3}$ titers for E monomer and E dimer, respectively) and ED12 carrying the W101A mutation (e; $9.43 \times 10^{3}$ and $4.84 \times 10^{3}$ titers for E monomer and E dimer, respectively), as well as DENV2 ED12 (f; $5.92 \times 10^{3}$ and $1.12 \times 10^{3}$ titers for $E$ monomer and $E$ dimer, respectively). In all cases, $n=6$ individual mouse serum samples per group. Data are presented as individual data points and arithmetic means (solid lines). In e, profiles obtained with the wild-type ZIKV ED12 (dashed lines) are included for comparison. $\mathbf{g}$, Serum was depleted of specific antibodies using antigen-displaying yeast, then analyzed for ZIKV neutralization activity by FRNT assay. FRNT 50 titers were calculated (for each group, $n=2$ independent depletion experiments using pooled sera). The data are presented as floating bars showing the mean (central line), spread and individual measurements for each group and depletion. $\mathbf{h}$, Infection enhancement curves of U937 cells infected with DENV2 16681 in the presence of E-monomer immune mouse serum after the depletion of specific antibodies as in $\mathbf{g}$ (for each depletion, $n=2$ independent experiments using depleted pooled sera from $\mathbf{h}$ ). Data are shown as single data points and arithmetic means (solid and dashed lines).

activity intact. This indicates that the majority of ZIKV E-monomer neutralizing activity resides in ED12. The low neutralizing activity of anti-ED3 antibodies was unexpected given the strong antiED3 responses observed in both groups of immunized mice ${ }^{14,40}$.
Depletion of E-monomer-reactive antibodies from E-dimerimmunized sera leaves most of the neutralizing activity intact, showing that E-dimer immunization induces antibodies to quaternary epitopes found only on the E dimer, which can neutralize 

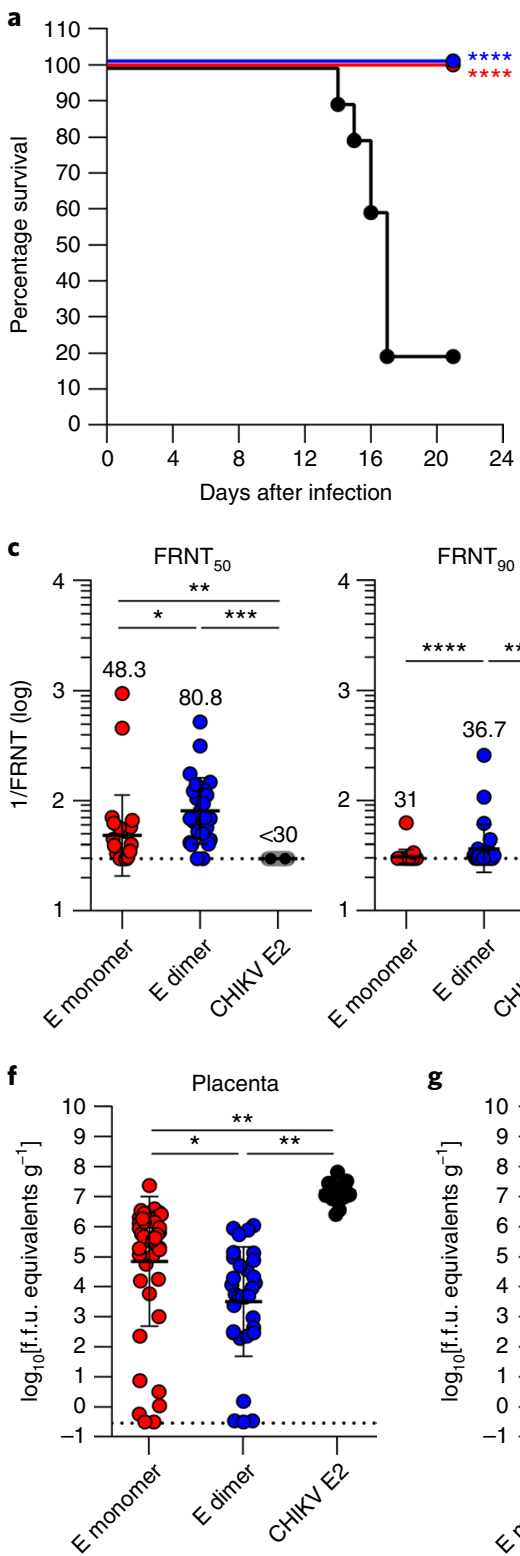

b

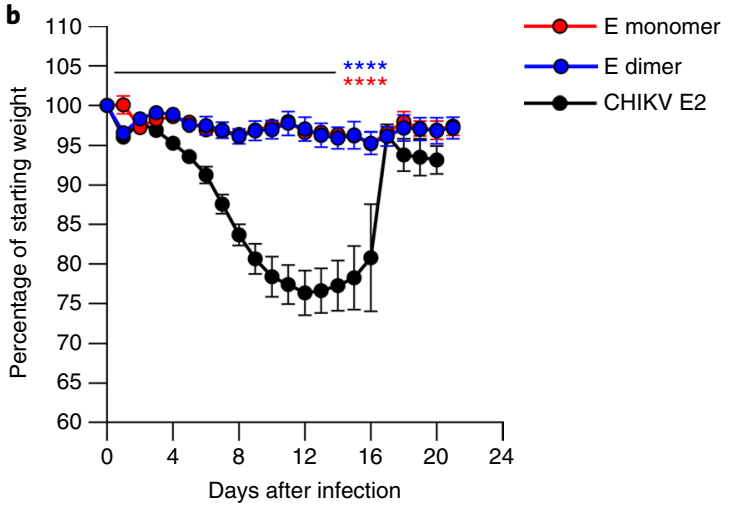

d

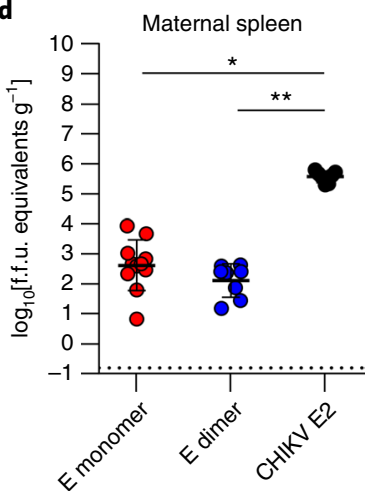

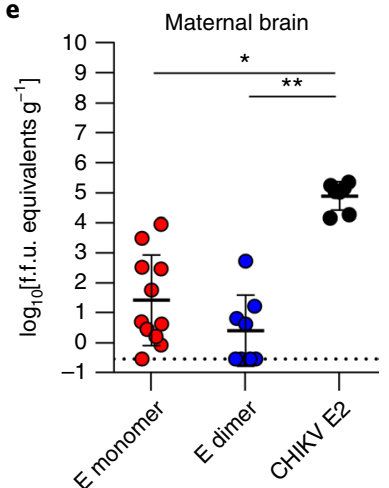

g

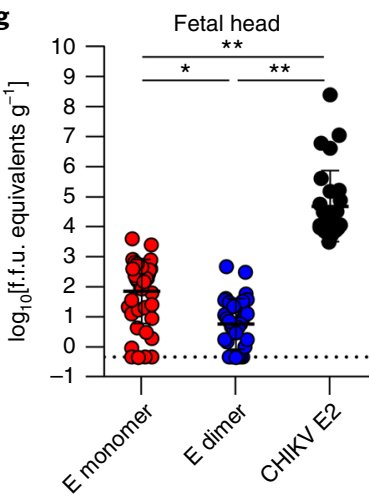

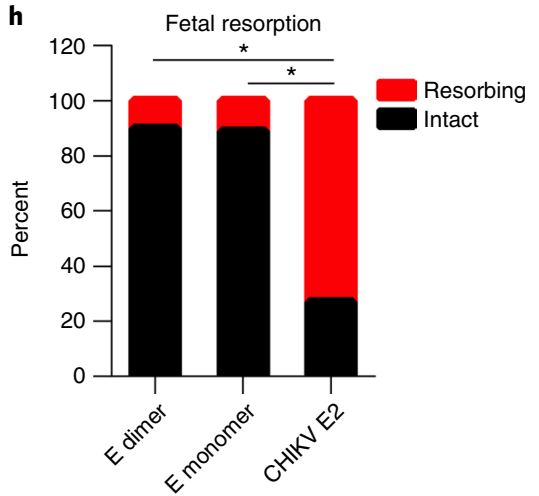

Fig. 5 | The E-dimer vaccine protects against ZIKV infection in C57BL/6 mice. a,b, Survival (a) and weight loss data (b) obtained from immunized wildtype C57BL/6 mice ( $n=10$ animals from two independent experiments) after challenge with ZIKV Dakar 41519. Survival data were analyzed by two-sided log-rank test. Weight loss was analyzed by Tukey-Kramer multiple comparison test for surviving animals. In $\mathbf{b}$, the line indicates days with statistically significant differences compared with the CHIKV E2 vaccine, and data are presented as means \pm s.d. c, ZIKV neutralization activity of serum collected 12 weeks post-prime. EC $_{50}$ (effective concentration of half-maximal inhibition, 1/FRNT ${ }_{50}$; left) and EC Ev $_{90}$ values (1/FRTN $_{90}$; right) against ZIKV were calculated for individual animals in each group. The dashed lines indicate the limit of detection of the assay. Data were analyzed by Kruskal-Wallis test with Dunn's post-test. Individual measurements, as well as geometric mean \pm s.d. values are noted ( $n=25$ individual mouse serum samples per group; 15 females and 10 males). d-g, ZIKV burden of immunized dams challenged with ZIKV Dakar 41525. Results are given for the maternal tissues (spleen (d) and brain (e); $n=7,11$ and 8 animals for the CHIKV E2, E-monomer and E-dimer groups, respectively), placenta (f; $n=28,44$ and 32 samples for the CHIKV E2, E-monomer and E-dimer groups, respectively) and fetal head ( $\mathbf{g} ; n=28,44$ and 32 samples for the CHIKV E2, E-monomer and E-dimer groups, respectively) harvested on E13. Data were analyzed by Kruskal-Wallis analysis of variance with Dunn's post-test. Solid bars denote median values, and dashed lines mark the limit of detection of each assay. $\mathbf{h}$, Fetal resorption was assessed at the time of harvest ( $n=79$, 105 and 68 samples for the CHIKV E2, E-monomer and E-dimer groups, respectively). The data were analyzed by chi-squared test. In all panels with $P$ values: ${ }^{\star} P<0.05$; ${ }^{\star \star} P<0.01$; $\star \star \star P<0.001 ; * \star \star \star P<0.0001$.

ZIKV. As expected, after further depletion with E-dimer protein, the neutralizing activity was lost from the E-dimer-immune serum.

We tested the ADE potential of ZIKV E-monomer-immune serum on DENV2 after depletion (Fig. 4h). Depletion with ZIKV ED12 or ED123, or DENV2 ED12 (all of which deplete FLE-reactive antibodies) almost completely removed ADE activity. However, depletion with ZIKV ED12 or ED123 W101A mutant yeasts para- doxically increased the enhancement of DENV2 ADE. We believe that this is because these constructs deplete all neutralizing activity from serum, leaving a residual antibody fraction that binds to the FLE and is poorly neutralizing but strongly enhancing. Notably, a separate set of $\mathrm{BALB} / \mathrm{c}$ mice, immunized with the same proteins but using the adjuvant Alum instead, replicated the antibody responses obtained with AddaVax (Supplementary Fig. 3). 
Next, we tested for protection from ZIKV challenge in C57BL/6 wild-type mice immunized with either E dimers, E monomers or the control CHIKV E2 protein. To create a lethal challenge model, we passively transferred $2 \mathrm{mg}$ of a type I interferon receptor (Ifnar1)blocking $\mathrm{mAb}^{41} 1 \mathrm{~d}$ before infection with $10^{5}$ focus-forming units (f.f.u.) of a mouse-adapted African ZIKV strain (Dakar 41519) ${ }^{24,42}$. All mice immunized with E-monomer or E-dimer proteins were protected against lethal ZIKV infection compared with the control group, which had a $20 \%$ survival rate (Fig. 5a). Mice vaccinated with the E-monomer or E-dimer proteins were similarly protected from clinical illness, as quantified by weight loss, compared with the CHIKV E2 control group (Fig. 5b). The ZIKV-neutralizing activity (half-maximum effective concentration $\left(\mathrm{EC}_{50}\right)$ titers of 80.8 and 48.3, and $\mathrm{EC}_{90}$ titers of 36.7 and 31.0, respectively, for the $\mathrm{E}$ dimer versus the $\mathrm{E}$ monomer) was significantly higher in the E-dimer group compared with E-monomer-immunized mice (Fig. $5 \mathrm{c}$ and Supplementary Fig. 4)

Next, we evaluated the efficacy of the E-protein vaccines against ZIKV in pregnancy. At approximately day 105 after initial vaccination, immunized mice were mated with wild-type sires. Five days later (embryo day 5 (E5)), dams were treated with the anti-Ifnar1 blocking $\mathrm{mAb}$, which allows ZIKV to disseminate to the placenta? One day later (at E6), dams were challenged subcutaneously with an African ZIKV strain ${ }^{43}$. On E13 (7 d post-infection), ZIKV RNA levels in maternal and fetal tissues were measured by real-time quantitative reverse transcription PCR. Viral RNA was substantially reduced in the spleen (approximately three $\log _{10}$ reductions; Fig. $5 \mathrm{~d}$ ) and brain (greater than four $\log _{10}$ reductions; Fig. 5e) of maternal tissues from mice vaccinated with $\mathrm{E}$ monomer or $\mathrm{E}$ dimer, which contrasts with observations in CHIKV E2-immunized mice.

We assessed the ZIKV burden in the placentas and fetuses of vaccinated mice. Placental tissues from E-monomer- and E-dimervaccinated dams showed a marked reduction in viral burden compared with CHIKV E2-vaccinated dams, with the E-dimer vaccine showing better protection (3.4 versus $1.5 \log$ reductions for the $\mathrm{E}$ dimer versus the E monomer, respectively; Fig. 5f). Analysis of fetal heads isolated from vaccinated dams also showed protection against vertical transmission, with substantial reductions in the burden of ZIKV RNA (Fig. 5g); again, the E-dimer vaccine performed better than the E-monomer vaccine, with 23-fold less ZIKV RNA observed in fetal heads. Whereas only $27 \%$ of fetuses were morphologically intact in CHIKV E2-immunized dams, 90 and $89 \%$ of fetuses in E-dimer- and E-monomer-immunized dams, respectively, were intact (Fig. 5h). Thus, the E-dimer protein vaccine was protective against vertical ZIKV transmission and fetal demise in this stringent-challenge model of pregnant immunocompromised mice and, based on virological analysis, performed better than the E-monomer vaccine.

Vaccines against flaviviruses have been pursued for nearly 80 years, with the most notable success being the development of the yellow fever 17D vaccine by Max Thelier in the 1930s, which is still used today ${ }^{44}$. Effective vaccines against Japanese encephalitis and tick-borne encephalitis are also licensed for use ${ }^{45}$. One of the major challenges for vaccine development against DENV is the phenomena of ADE. This mandates that a safe DENV vaccine must achieve balanced and long-lasting neutralizing activity to all four DENV serotypes. Failure to achieve this may leave vaccinated individuals exposed to enhanced disease on natural infection. These limitations have meant that Dengvaxia-the first licensed DENV vaccine-is now recommended exclusively for DENV-immune but not DENV-naive individuals ${ }^{22}$.

ZIKV and the four DENV serotypes are closely related; pairwise comparisons between the ZIKV and DENV E proteins reveal 55$58 \%$ sequence identity, whereas pairwise comparisons of the four DENV E proteins reveals 65-70\% sequence identity ${ }^{12,46}$. Thus, at the genetic level, ZIKV is not that much different from DENV than the four serotypes of DENV are from each other. The similarity between ZIKV and the DENV means there is substantial cross-reactivity of the serological responses to the two viruses. Interestingly, we found that the cross-reactivity between ZIKV E-monomer-immune serum and DENV was variable, with the least cross-reactivity to DENV4, which may be partly explained by their phylogenetic distance ${ }^{47}$.

As ZIKV and DENV are both transmitted by the same insect vector (usually Aedes Aegypti $i^{6}$ ), populations can be at risk of infection by both viruses. The South American epidemic of ZIKV in 2015 occurred in the background of high DENV seroprevalence. Interaction between the viruses in terms of antibodies, with immunity to one promoting ADE of the other, is a concern, even though the epidemiological evidence to date has not defined this relationship $^{20,21}$. Nonetheless, there are some indications that reciprocal ADE could occur in humans ${ }^{48}$. Designing effective vaccines that minimize the risk of ADE between ZIKV and DENV would mitigate such concerns.

We show here that ZIKV E dimers produced by mammalian expression minimize exposure of the FLE and maintain the EDE. The ZIKV E-dimer subunit vaccine has the advantage that it does not contain precursor membrane protein or expose the FLE-two immunodominant antigens that are recognized by enhancing antibodies $^{1-3}$. Stable ZIKV E dimers are immunogenic, and protect against ZIKV challenge and infection of the placenta and fetus in pregnant mice. As responses to the FLE are minimized, crossreactivity to DENV is limited, which markedly reduces the ability of ZIKV E-dimer vaccination to prime for ADE of DENV. Previous studies have introduced FLE mutations to reduce ADE. These mutations significantly reduced heterotypic DENV ADE of infection in ZIKV-immunized mice, but at the expense of a significant reduction in neutralizing titers compared with the unmutated construct $^{49}$. Mutation of the FLE destroys the EDE and may also disrupt dimer formation, therefore preventing the induction of EDE and other dimer-dependent antibodies. While we used recombinant, HEK293T cell-produced, stable E-dimer protein as the immunogen, it could also be used with alternative methods of immunization, including messenger RNA vaccination or DNA plasmid vaccination, to induce high titer antibody responses in mice ${ }^{49}$.

We did not find evidence that ZIKV E-dimer immunization could induce quaternary E-dimer-specific antibodies that crossreact to the EDE on the DENV. In DENV, sequential natural infections with different DENV serotypes may drive the evolution of broadly cross-reactive EDE-specific antibodies. Our future experiments will test the feasibility of generating such DENV/ZIKV EDE-specific antibodies using sequential E-dimer prime-boosting approaches with heterologous antigens.

\section{Online content}

Any methods, additional references, Nature Research reporting summaries, source data, statements of code and data availability and associated accession codes are available at https://doi.org/10.1038/ s41590-019-0477-Z.

Received: 6 June 2019; Accepted: 22 July 2019; Published online: 2 September 2019

\section{References}

1. Dejnirattisai, W. et al. Cross-reacting antibodies enhance dengue virus infection in humans. Science 328, 745-748 (2010).

2. Beltramello, M. et al. The human immune response to dengue virus is dominated by highly cross-reactive antibodies endowed with neutralizing and enhancing activity. Cell Host Microbe 8, 271-283 (2010).

3. Stiasny, K., Kiermayr, S., Holzmann, H. \& Heinz, F. X. Cryptic properties of a cluster of dominant flavivirus cross-reactive antigenic sites. J. Virol. 80, 9557-9568 (2006).

4. Dejnirattisai, W. et al. A new class of highly potent, broadly neutralizing antibodies isolated from viremic patients infected with dengue virus. Nat. Immunol. 16, 170-177 (2015). 
5. Halstead, S. B. \& O’Rourke, E. J. Antibody-enhanced dengue virus infection in primate leukocytes. Nature 265, 739-741 (1977).

6. Musso, D. \& Gubler, D. J. Zika virus. Clinical Microbiol. Rev. 29, 487-524 (2016)

7. Cao-Lormeau, V. M. et al. Guillain-Barre syndrome outbreak associated with Zika virus infection in French Polynesia: a case-control study. Lancet 387, 1531-1539 (2016)

8. Brasil, P. et al. Zika virus outbreak in Rio de Janeiro, Brazil: clinical characterization, epidemiological and virological aspects. PLoS Negl. Trop. Dis. 10, e0004636 (2016).

9. Miner, J. J. et al. Zika virus infection during pregnancy in mice causes placental damage and fetal demise. Cell 165, 1081-1091 (2016).

10. Diamond, M. S., Ledgerwood, J. E. \& Pierson, T. C. Zika virus vaccine development: progress in the face of new challenges. Annu. Rev. Med. 70, 121-135 (2019).

11. Crill, W. D. \& Chang, G. J. Localization and characterization of flavivirus envelope glycoprotein cross-reactive epitopes. J. Virol. 78, 13975-13986 (2004).

12. Dejnirattisai, W. et al. Dengue virus sero-cross-reactivity drives antibodydependent enhancement of infection with Zika virus. Nat. Immunol. 17, 1102-1108 (2016).

13. Barba-Spaeth, G. et al. Structural basis of potent Zika-dengue virus antibody cross-neutralization. Nature 536, 48-53 (2016).

14. Stettler, K. et al. Specificity, cross-reactivity, and function of antibodies elicited by Zika virus infection. Science 353, 823-826 (2016).

15. Bardina, S. V. et al. Enhancement of Zika virus pathogenesis by preexisting antiflavivirus immunity. Science 356, 175-180 (2017).

16. Brown, J. A. et al. Dengue virus immunity increases Zika virus-induced damage during pregnancy. Immunity 50, 751-762 (2019).

17. McCracken, M. K. et al. Impact of prior flavivirus immunity on Zika virus infection in rhesus macaques. PLoS Pathog. 13, e1006487 (2017).

18. George, J. et al. Prior exposure to Zika virus significantly enhances peak dengue-2 viremia in rhesus macaques. Sci. Rep. 7, 10498 (2017).

19. Pantoja, P. et al. Zika virus pathogenesis in rhesus macaques is unaffected by pre-existing immunity to dengue virus. Nat. Commun. 8, 15674 (2017).

20. Gordon, A. et al. Prior dengue virus infection and risk of Zika: a pediatric cohort in Nicaragua. PLoS Med. 16, e1002726 (2019)

21. Rodriguez-Barraquer, I. et al. Impact of preexisting dengue immunity on Zika virus emergence in a dengue endemic region. Science 363, 607-610 (2019).

22. Rey, F. A., Stiasny, K., Vaney, M. C., Dellarole, M. \& Heinz, F. X. The brigh and the dark side of human antibody responses to flaviviruses: lessons for vaccine design. EMBO Rep. 19, 206-224 (2018).

23. De Alwis, R. et al. Identification of human neutralizing antibodies that bind to complex epitopes on dengue virions. Proc. Natl Acad. Sci. USA 109 7439-7444 (2012).

24. Zhao, H. et al. Structural basis of Zika virus-specific antibody protection. Cell 166, 1016-1027 (2016)

25. Rouvinski, A. et al. Recognition determinants of broadly neutralizing human antibodies against dengue viruses. Nature 520, 109-113 (2015).

26. Abbink, P. et al. Therapeutic and protective efficacy of a dengue antibody against Zika infection in rhesus monkeys. Nat. Med. 24, 721-723 (2018).

27. Fernandez, E. et al. Human antibodies to the dengue virus E-dimer epitope have therapeutic activity against Zika virus infection. Nat. Immunol. 18 1261-1269 (2017).

28. Teoh, E. P. et al. The structural basis for serotype-specific neutralization of dengue virus by a human antibody. Sci. Transl. Med. 4, 139ral83 (2012).

29. Fibriansah, G. et al. A highly potent human antibody neutralizes dengue virus serotype 3 by binding across three surface proteins. Nat. Commun. 6, 6341 (2015).

30. Hasan, S. S. et al. A human antibody against Zika virus crosslinks the $\mathrm{E}$ protein to prevent infection. Nat. Commun. 8, 14722 (2017).

31. Fibriansah, G. et al. Cryo-EM structure of an antibody that neutralizes dengue virus type 2 by locking E protein dimers. Science 349, 88-91 (2015)

32. Long, F. et al. Structural basis of a potent human monoclonal antibody against Zika virus targeting a quaternary epitope. Proc. Natl Acad. Sci. USA 116, 1591-1596 (2019).

33. Wang, J. et al. A human bi-specific antibody against Zika virus with high therapeutic potential. Cell 171, 229-241 (2017).

34. Bailey, M. J. et al. Human monoclonal antibodies potently neutralize Zika virus and select for escape mutations on the lateral ridge of the envelope protein. J. Virol. 93, e00405-e00419 (2019).

35. Rouvinski, A. et al. Covalently linked dengue virus envelope glycoprotein dimers reduce exposure of the immunodominant fusion loop epitope. Nat. Commun. 8, 15411 (2017).
36. Slon Campos, J. L. et al. Temperature-dependent folding allows stable dimerization of secretory and virus-associated $\mathrm{E}$ proteins of dengue and Zika viruses in mammalian cells. Sci. Rep. 7, 966 (2017).

37. Pierson, T. C. et al. The stoichiometry of antibody-mediated neutralization and enhancement of West Nile virus infection. Cell Host Microbe 1, 135-145 (2007).

38. Oliphant, T. et al. Induction of epitope-specific neutralizing antibodies against West Nile virus. J. Virol. 81, 11828-11839 (2007)

39. Collins, M. H. et al. Lack of durable cross-neutralizing antibodies against Zika virus from dengue virus infection. Emerg. Infect. Dis. 23, 773-781 (2017)

40. Robbiani, D. F. et al. Recurrent potent human neutralizing antibodies to Zika virus in Brazil and Mexico. Cell 169, 597-609 (2017).

41. Sheehan, K. C. et al. Blocking monoclonal antibodies specific for mouse IFN- $\alpha / \beta$ receptor subunit 1 (IFNAR-1) from mice immunized by in vivo hydrodynamic transfection. J. Interferon Cytokine Res. 26, 804-819 (2006).

42. Sapparapu, G. et al. Neutralizing human antibodies prevent Zika virus replication and fetal disease in mice. Nature 540, 443-447 (2016).

43. Gorman, M. J., Poddar, S., Farzan, M. \& Diamond, M. S. The interferonstimulated gene Ifitm 3 restricts West Nile virus infection and pathogenesis. J. Virol. 90, 8212-8225 (2016).

44. Frierson, J. G. The yellow fever vaccine: a history. Yale J. Biol. Med. 83, 77-85 (2010).

45. Collins, M. H. \& Metz, S. W. Progress and works in progress: update on flavivirus vaccine development. Clin. Ther. 39, 1519-1536 (2017).

46. Flipse, J. \& Smit, J. M. The complexity of a dengue vaccine: a review of the human antibody response. PLoS Negl. Trop. Dis. 9, e0003749 (2015).

47. Keasey, S. L. et al. Antibody responses to Zika virus infections in environments of flavivirus endemicity. Clin. Vaccine Immunol. 24, e00036-17 (2017).

48. Langerak, T. et al. The possible role of cross-reactive dengue virus antibodies in Zika virus pathogenesis. PLoS Pathog. 15, e1007640 (2019).

49. Richner, J. M. et al. Modified mRNA vaccines protect against Zika virus infection. Cell 168, 1114-1125 (2017).

\section{Acknowledgements}

This work was supported by the Wellcome Trust (collaborative grant 203224/Z/16/Z to G.R.S. and J.M.), National Institute for Health Research Biomedical Research Centre Funding Scheme (to G.R.S.), Medical Research Council Newton fund (UK-Thailand joint initiative of infectious diseases grant MR/R020957/1 to J.M.), National Institutes of Health (grants R01 AI073755, R01 AI127828 and R01 HD091218 to M.S.D., and grant T32 AI007172 to B.W.J.), UK Department of Health and Social Care (projects 972216 and 971557 to A.R.-S.) and French Government's Programme d'Investissements d'Avenir (Labex Integrative Biology of Emerging Infectious Diseases grant ANR-10-LABX-62IBEID 4E AAP to F.A.R.). G.R.S. is supported as a Wellcome Trust Senior Investigator (grant 095541/A/11/Z). We thank Y. C. Kim and B. D. Jimenez for technical assistance.

\section{Author contributions}

G.R.S., J.M. and F.A.R. conceived the study. G.R.S., J.M., F.A.R., M.S.D., A.R.-S., J.L.S.-C., W.D., B.W.J., L.A.D., E.S.W., R.E.C. and C.L.-C. designed the experiments and analyzed the data. J.L.S.-C., W.D., B.W.J., C.L.-C. and W.W. performed the experiments. G.R.S. wrote the paper, with editorial input from all co-authors.

\section{Competing interests}

G.R.S., F.A.R. and J.M. are named inventors on a patent owned by Imperial College London and Institut Pasteur describing the EDE and its use as a potential immunogen (application number EP3172229A1). G.R.S. sits on the advisory board of GSK Vaccines. M.S.D. is a consultant for Inbios and Atreca, and sits on the Scientific Advisory Board at Moderna. The remaining authors declare no competing interests.

\section{Additional information}

Supplementary information is available for this paper at https://doi.org/10.1038/ s41590-019-0477-z.

Reprints and permissions information is available at www.nature.com/reprints. Correspondence and requests for materials should be addressed to J.M. or G.R.S.

Peer review information: Jamie Wilson was the primary editor on this article and managed its editorial process and peer review in collaboration with the rest of the editorial team.

Publisher's note: Springer Nature remains neutral with regard to jurisdictional claims in published maps and institutional affiliations.

(c) The Author(s), under exclusive licence to Springer Nature America, Inc. 2019 


\section{Methods}

Cells, antibodies and reagents. HEK293T cells, Vero cells (a gift from the Armed Forces Research Institute of Medical Sciences (AFRIMS)) and U937 cells were cultured at $37^{\circ} \mathrm{C}$ in DMEM (D6046; Sigma-Aldrich), MEM (31095; Gibco) and RPMI-1640 (R8758; Sigma-Aldrich), respectively. C6/36 cells (a gift from AFRIMS) were grown in Leibovitz L-15 (11415; Gibco) at $28^{\circ} \mathrm{C}$. Media was supplemented with $10 \%$ heat-inactivated fetal bovine serum (10270106; Gibco),

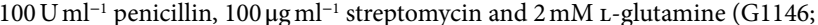
Sigma-Aldrich). All cell lines were free from mycoplasma contamination.

Horseradish peroxidase (HRP)-conjugated anti-mouse immunoglobulin G (IgG) (5220-0343; lot 10246668; 1:10,000) was purchased from SeraCare. Fluorescein isothiocyanate (FITC)-conjugated anti-human Ig (F1085; lot 0095473; 1:200) and FITC-conjugated anti-mouse Ig (F0479; lot 20047943; 1:200) were purchased from Dako. Alkaline phosphatase (ALP)-conjugated anti-mouse IgG + IgM (A9316; lot 061M6260; 1:2,000) and ALP-conjugated anti-human IgG (A9544; lot 026M4768; 1:5,000) were purchased from Sigma-Aldrich. Anti-Xpress Antibody (R910-25; lot 1833004; 1:5,000) was purchased from Life Technologies. Anti-mouse Ifnarl (Clone MAR1-5A3; I-1188; $10 \mathrm{mg} \mathrm{ml}^{-1}$ ) was purchased from Leinco. The mouse monoclonal anti-DENV E 4G2 was a gift from AFRIMS. Monoclonal human anti-EDE1 (753-3C10; 752-2C8), anti-ED3 (ZA04-P4C5; ZA04-P5C10) and anti-FLE (758-P6B10; 759-B12) antibodies, as well as mouse anti-SV5 and human anti-SV5 (simian virus 5-derived tag (sequence GKPIPNPLLGLD)) and anti-roTag $(1 \mathrm{~F} 2)^{50}$ antibodies were produced as recombinant proteins and purified using Protein A/G-conjugated agarose beads from polyethylenimine (PEI)-transfected HEK293T cells. The DNA constructs required to produced anti-SV 5 and anti-roTag $m A$ bs were provided by $O$. R. Burrone (International Centre for Genetic Engineering and Biotechnology, Trieste, Italy).

PFHM-II Protein-Free Hybridoma Medium (12040077) was purchased from Life Technologies. Bovine serum albumin (BSA; A7030), SIGMAFAST p-nitrophenyl phosphate tablets (pNPP; N2770), PEI (MW: 25,000; branched: 408,727 ) and 3,3'-diaminobenzidine tetrahydrochloride tablets (DAB; D5905) were purchased from Sigma-Aldrich. Yeast Nitrogen Base media without amino acids (YNB; 291940) and Casamino acids (CAA; 223050) were purchased from BD Biosciences. Amphotericin B (Fungizone; 15290026) was purchased from Gibco. The adjuvants AddaVax (vac-adx-10) and Alum (Alhydrogel; vac-alu-250) were purchased from InvivoGen. Protein G/Protein A agar suspension beads (IP10; $10 \mathrm{ml}$ ) and Luminata Classico Western Blot developer solution (WBLUC0500) were obtained from Merck Chemicals. CNBr-activated sepharose 4B beads (17-0430-01) and nitrocellulose membrane (10600004) were purchased from GE Healthcare. Quick Blue protein strain solution was purchased from Avidity Science. Phusion DNA polymerase (M0530) and Taq DNA ligase (M0208) were purchased from New England BioLabs) 5'-T5 exonuclease (T5E4111K) was purchased from Epicentre.

Viral stocks. ZIKV strain PF13/251013-18 (PF13) was isolated from a patient during the ZIKV outbreak in French Polynesia in 2013. ZIKV Dakar strain 41525 (Senegal, 1984; GenBank accession number KU955591) was acquired from the World Reference Center for Emerging Viruses and Arboviruses. The mouse-adapted ZIKV Dakar strain was previously described ${ }^{51}$. DENV1 (Hawaii), DENV2 (16681) and DENV3 (H87) were gifts from AFRIMS. DENV4 (1-0093) was isolated from a DENV4-infected patient. Except for ZIKV Dakar, which was propagated in Vero cells, all viruses were produced in C6/36 Aedes albopictus cells. Virus-containing supernatants were clarified by centrifugation at 3,000 r.p.m. at $4{ }^{\circ} \mathrm{C}$ before being stored at $-80^{\circ} \mathrm{C}$. Viral titers were determined by focusforming assays. Briefly, tenfold serial dilutions of virus stocks were added to Vero cell monolayers. Cells were then stained with the anti-E mAb 4G2 followed by peroxidase-conjugated goat anti-mouse Ig (1:1,000 dilution). The foci (infected cells) were visualized by adding the peroxidase substrate DAB on the Vero cells.

Gene sequences and plasmid DNA constructs. Synthetic fragments (obtained from GeneArt, Life Technologies) coding for the human codon-optimized ED123 sequences from the ZIKV strain PF13/251013-18 (PF13) (GenBank accession number KJ776791; E-protein amino acids 1-404) and the S. cerevisiae-optimized sequence from the DENV2 16681 strain (GenBank accession number U87411; E-protein amino acids 1-395) were obtained and used for subsequent cloning.

For production of the recombinant protein, the ZIKV PF13 ED123 sequence, including the M-derived secretion signal (final transmembrane domain of the membrane protein (M); sequence: VIYLVMILLIAPAYS), was cloned between an amino-terminal methionine and a carboxy-terminal SV5 (GKPIPNPLLGLD) in pVAX vectors (Life Technologies). For yeast display, the ED123 sequence (amino acids 1-404), or the ED12 (amino acids 1-302 for ZIKV; 1-296 for DENV2) and ED3 sequences (amino acids 303-404), obtained by PCR from the ZIKV PF13 or DENV2 16681 ED123 original gene were cloned into the PYD1 yeast display vector according to the manufacturer instructions (V835-01; Life Technologies).

Mutations to obtain covalent ZIKV E dimers (L107C, A264C and A319C) and to disrupt the FLE (W101A) were inserted using standard Gibson assembly cloning with primers containing the mutant sequence, and mutations were verified by DNA sequencing. The plasmid encoding the CHIKV E2 protein was obtained as previously described ${ }^{52}$
To avoid the potential serological interference of the SV5 tag present within the ZIKV antigens used for immunization, an alternatively tagged ZIKV E dimer was obtained by cloning the sequence encoding the triple-mutant ZIKV PF13 ED123 dimer into a similar pVax vector upstream of the roTag (GSGSISSSIFKNEG) $\operatorname{tag}^{50}$, as described above.

Expression and purification of recombinant proteins. DNA plasmids encoding the SV5-tagged ZIKV E-monomer and E-dimer proteins were transfected into HEK293T cells by PEI-mediated transfection. After overnight incubation at $37^{\circ} \mathrm{C}$, the culture medium was replaced with a protein-free medium, and the cells were incubated for another $6 \mathrm{~d}$ at $28^{\circ} \mathrm{C}^{36}$. Supernatants were then collected and cleared by centrifugation followed by filtration, and protein expression was assessed by Western blotting (below) before purification. ZIKV E monomers and E dimers were affinity-purified using anti-SV5-conjugated sepharose beads, followed by SEC (Superdex 200 10/300 GL column; GE Life Sciences) in PBS to remove aggregates. The purity of the proteins was assessed by reducing $(10 \% \beta$-mercaptoethanol ( $\beta$ $\mathrm{ME})$ ) and non-reducing sodium dodecyl sulfate polyacrylamide gel electrophoresis (SDS-PAGE) ( $\sim 3 \mu \mathrm{g}$ of protein) and SEC ( $150 \mu \mathrm{g}$ of protein), as before, until clean bands and peaks were obtained. CHIKV E2 protein was produced, purified and tested as previously described ${ }^{52}$. Purified proteins were concentrated in PBS, quantified by spectrophotometry, sterilized by filtration (Spin-X tube filter; 8160 Costar) and kept at $-80^{\circ} \mathrm{C}$ until use.

The RoTag-tagged ZIKV E dimer used for the analysis of immunized mouse serum was expressed as recombinant protein in HEK293T cells, as described before; culture supernatants containing the protein were clarified by centrifugation, filtered and kept at $-80^{\circ} \mathrm{C}$ until use.

Expression of human mAbs against DENV E. Pairs of plasmids encoding the heavy and light chain of human IgG1 were transfected in HEK293T as described above and harvested after $5 \mathrm{~d}$. The antibody concentration was measured by ELISA and samples were stored at $-20^{\circ} \mathrm{C}$.

Immunoblotting. Samples were separated by non-reducing or reducing (10\% $\beta$-ME) SDS-PAGE, transferred to nitrocellulose membranes and blocked with a $4 \%$ milk solution in PBS. Membranes were incubated with anti-SV5 $\mathrm{mAb}\left(1 \mu \mathrm{g} \mathrm{ml}^{-1}\right)$ for $1 \mathrm{~h}$, washed, and probed with HRP-linked anti-mouse IgG goat antibodies $(1: 1,000)$ for $1 \mathrm{~h}$. Signals were developed using Luminata Classico Western HRP substrate.

ELISA analysis of purified proteins. Nunc MaxiSorp immunoplates (442404; Thermo Fisher Scientific) were coated with $50 \mu \mathrm{l}$ of $5 \mu \mathrm{g} \mathrm{ml}^{-1}$ of human anti-SV5. The plate was then blocked with PBS and 2\% BSA for $1 \mathrm{~h}$, followed by incubation with $50 \mu \mathrm{l}$ of a $20 \mathrm{ng} \mathrm{ml}^{-1}$ solution of purified ZIKV E monomer and E dimer in PBS and $2 \%$ BSA for $1 \mathrm{~h}$. Plates were then incubated with $50 \mu \mathrm{l}$ of anti-FLE and anti-EDE $\mathrm{mAb}$ supernatants $\left(2 \mu \mathrm{g} \mathrm{ml}^{-1}\right)$, followed by ALP-conjugated anti-human $\operatorname{IgG}(1: 10,000)$. The assays were developed with $\mathrm{pNPP}$ reagent for $1 \mathrm{~h}$ and measured at $405 \mathrm{~nm}$.

Animal experiments. For the assessment of immunogenicity, 6- to 8-week-old female inbred BALB/c (H-2d) mice and 6- to 8-week-old female outbred CD1 mice were purchased from Envigo. For the viral challenge experiments, 6- to 8-weekold male and female C57BL/6J wild-type (000064) mice were purchased from Jackson Laboratories. Animals were immunized three times at 4-week intervals by intramuscular injection (hind leg) of $10 \mu \mathrm{g}$ of antigen in a $50 \mu \mathrm{l}$ total volume with $50 \% v / v$ of adjuvant (AddaVax or Alum). For the assessment of immunogenicity, blood samples were collected by tail bleeding 4 weeks after priming and then at 2 -week intervals. Serum samples were collected, heat inactivated $\left(56^{\circ} \mathrm{C}\right.$ for $\left.30 \mathrm{~min}\right)$ and stored at $-20^{\circ} \mathrm{C}$ until use.

The anti-Ifnar $1 \mathrm{mAb}$ challenge model was utilized as previously described ${ }^{53}$. Briefly, male mice were injected with $2 \mathrm{mg}$ anti-Ifnar $1 \mathrm{mAb}$ (MAR1-5A3) followed by subcutaneous footpad challenge with $10^{5}$ f.f.u. mouse-adapted Dakar ZIKV $1 \mathrm{~d}$ later ${ }^{51}$. The mice were weighed and their mortality was assessed daily. The antiIfnar $1 \mathrm{mAb}$ pregnancy challenge model was previously described ${ }^{9,49}$. Briefly, $5 \mathrm{~d}$ after the identification of a copulation plug, $2 \mathrm{mg}$ anti-Ifnar1 mAb (MAR1-5A3) was administered by intraperitoneal injection to female mice, followed $1 \mathrm{~d}$ later by infection with $10^{5}$ f.f.u. mouse-adapted Dakar ZIKV by subcutaneous footpad injection. Pregnant mice were harvested $7 \mathrm{~d}$ after infection, and viral burdens were determined via real-time quantitative reverse transcription PCR as previously described 9 .

ELISA analysis of immunized mouse sera. For the virus ELISA, Nunc MaxiSorp immunoplates were coated with $50 \mu \mathrm{l}$ of $5 \mu \mathrm{g} \mathrm{ml}^{-1}$ of a human anti-FLE antibody isolated from a DENV-infected patient (mAb 758-P6B10)4. The plate was blocked with PBS and 2\% BSA for $1 \mathrm{~h}$, followed by incubation with ZIKV PF13 viral supernatant for $1 \mathrm{~h}$. Non-infected C6/36 cell-cultured supernatant was used as mock control. For ZIKV E-dimer ELISA, immunoplates were coated with $50 \mu$ of $5 \mathrm{\mu g} \mathrm{ml}^{-1}$ of a human anti-roTag $(\mathrm{mAb} 1 \mathrm{~F} 2)^{50}$, followed by blocking and $1 \mathrm{~h}$ incubation with $50 \mu \mathrm{l}$ of HEK293T cell-culture supernatants containing roTagtagged ZIKV E dimers. Supernatant from mock-transfected cells was used as 
a background control. Plates were then incubated with $50 \mu$ of threefold serial dilutions of mouse sera in PBS and 2\% BSA, followed by ALP-conjugated antimouse IgG + IgM (1:2,000). The assays were developed with pNPP for $1 \mathrm{~h}$, stopped with $\mathrm{NaOH} 2 \mathrm{M}$, and measured at $405 \mathrm{~nm}$. Endpoint titers were defined as the reciprocal serum dilution that corresponded to three times the average optical density value obtained for the negative control, after subtraction of the average background.

Yeast display and flow cytometry. pYD1 plasmids containing a TRP1 selectable marker and encoding the ZIKV and DENV2 E-derived protein antigens were transformed into the $S$. cerevisiae strain EBY100. Protein expression was induced by culture in glucose-deprived YNB-CAA media with $2 \%$ galactose at $20^{\circ} \mathrm{C}$ for $30 \mathrm{~h}$. Cultures were harvested, and stable clones were selected and isolated by surface staining with both anti-Xpress-FITC antibody (R910-25; Invitrogen) and the corresponding ZIKV/DENV anti-E protein antibodies by fluorescenceactivated cell sorting analysis. Antigen-expressing yeast cells were then resuspended in PBS and $20 \%$ glycerol and kept at $-80^{\circ} \mathrm{C}$ until use.

To assess the binding of mouse immune sera, thawed and washed yeast cells were incubated with twofold dilutions of mouse serum in PBS and $2 \%$ BSA for $1 \mathrm{~h}$ at $4{ }^{\circ} \mathrm{C}$, followed by incubation with FITC-conjugated anti-mouse Ig. Reactivity profiles were obtained using a BD LSRFortessa X-20 flow cytometer (BD Biosciences). Since a variable fraction of non-displaying yeasts remained on each batch and the surface protein display differed significantly depending on the constructs and the batch, serum reactivity profiles were normalized by taking the maximum protein display from each batch as $100 \%$ positive events. Reactivity titers were defined as the reciprocal serum dilution that corresponded to $50 \%$ of the maximum reactivity for each yeast. In all cases, the bulk yeast population was selected in the preliminary forward versus side scatter cell population plot, and boundaries between 'positive' and 'negative' populations in the 488-530_30A channel (for the FITC signal) were defined based on negative control cells (stained yeast cells without the primary antibody). Flow cytometry data are presented in the format of histograms to enforce the distinction between FITC-positive and FITCnegative cell populations.

Depletion assay. To prevent downstream contamination, yeasts were treated with amphotericin B $\left(25 \mu \mathrm{lml}^{-1}\right)$ overnight and washed before the assay. Each $1 \mathrm{ml}$ sample of pooled mouse serum, with a 1:100 dilution (in PBS and $2 \% \mathrm{BSA}$ ), was incubated with $500 \mu \mathrm{l}$ centrifuged antigen-displaying yeast for $2 \mathrm{~h}$ at $4{ }^{\circ} \mathrm{C}$. The depleted sera were tested for remaining reactivity, and additional rounds of depletion were performed until all of the antigen-specific activity was removed. Due to the low expression of the ED123 protein (with and without the W101A mutation), yeast expressing ED3 and ED12 (or ED12 W101A) was also added to ensure complete depletion. To deplete the fraction of E-dimer-reactive antibodies, ED123-depleted serum was further incubated with anti-roTag-coated sepharose beads, onto which roTag-tagged ZIKV E dimers had been captured. Depleted serum was then cleared by centrifugation, filter sterilized (Spin-X tube filter; 8160; Costar) and stored at $-20^{\circ} \mathrm{C}$.

Neutralization assay. The FRNT was performed by premixing virus with serially diluted mouse serum for $1 \mathrm{~h}$ at $37^{\circ} \mathrm{C}$. The mixtures were then added to Vero cells and incubated for 2 (for ZIKV) or $3 \mathrm{~d}$ (for DENV), and focus-forming assays were performed. The percentage focus reduction was calculated, and $50 \%$ and $90 \%$
FRNT measures were obtained using the probit model from the SPSS package or Prism linear regression.

ADE assay. Serially diluted mouse sera were incubated with virus at a multiplicity of infection of 2 for $1 \mathrm{~h}$ at $37^{\circ} \mathrm{C}$ before addition to U937 cells. After incubation for 2 (ZIKV) or $3 \mathrm{~d}$ (DENV), supernatants were harvested, and viral titers were determined by focus-forming assays.

Ethics. This study was carried out in accordance with the recommendations in the Guide for the Care and Use of Laboratory Animals of the National Institutes of Health and the UK Home Office Animals Act Project License. The protocols were approved by the Institutional Animal Care and Use Committee at the Washington University School of Medicine (assurance number: A3381-01), and by the University of Oxford Animal Care and Ethical Review Committee (PPL $30 / 2414)$. Virus inoculations were performed under anesthesia that was induced and maintained with ketamine hydrochloride and xylazine, and all efforts were made to minimize animal suffering.

Statistics and reproducibility. Depending on sample availability, data were obtained from at least two independent experiments performed in duplicate. When representative data are shown, the data were reproduced successfully and the number of independent repetitions is indicated. The two-sided log-rank test, Kruskal-Wallis test with Dunn's post-test, two-way analysis of variance followed by Tukey-Kramer multiple comparison test and chi-squared test were used in some experiments (GraphPad Prism 8.0; GraphPad Software). Sample size was not statistically assessed. No randomization was done. Investigators were not blinded for data collection or analysis.

For additional information regarding methods, reagents and reproducibility of the data shown here, please refer to the Reporting Summary published alongside this report.

Reporting Summary. Further information on research design is available in the Nature Research Reporting Summary linked to this article.

\section{Data availability}

The authors declare that the data and materials supporting the findings of this study are available within the paper and its Supplementary Information files, or can be obtained on request from the corresponding authors.

\section{References}

50. Petris, G., Bestagno, M., Arnoldi, F. \& Burrone, O. R. New tags for recombinant protein detection and O-glycosylation reporters. PLOS ONE 9, e96700 (2014).

51. Gorman, M. J. et al. An immunocompetent mouse model of Zika virus infection. Cell Host Microbe 23, 672-685 (2018).

52. Lopez-Camacho, C. et al. Assessment of immunogenicity and neutralisation efficacy of viral-vectored vaccines against chikungunya virus. Viruses 11, E322 (2019).

53. Lazear, H. M. et al. A mouse model of Zika virus pathogenesis. Cell Host Microbe 19, 720-730 (2016). 


\section{Reporting Summary}

Nature Research wishes to improve the reproducibility of the work that we publish. This form provides structure for consistency and transparency in reporting. For further information on Nature Research policies, see Authors \& Referees and the Editorial Policy Checklist.

\section{Statistics}

For all statistical analyses, confirm that the following items are present in the figure legend, table legend, main text, or Methods section.

$\mathrm{n} / \mathrm{a}$ Confirmed

$\bigotimes$ The exact sample size $(n)$ for each experimental group/condition, given as a discrete number and unit of measurement

\A statement on whether measurements were taken from distinct samples or whether the same sample was measured repeatedly

The statistical test(s) used AND whether they are one- or two-sided

Only common tests should be described solely by name; describe more complex techniques in the Methods section.

$\bigotimes$ A description of all covariates tested

$\square$ \ A description of any assumptions or corrections, such as tests of normality and adjustment for multiple comparisons

A full description of the statistical parameters including central tendency (e.g. means) or other basic estimates (e.g. regression coefficient)

AND variation (e.g. standard deviation) or associated estimates of uncertainty (e.g. confidence intervals)

For null hypothesis testing, the test statistic (e.g. $F, t, r$ ) with confidence intervals, effect sizes, degrees of freedom and $P$ value noted

Give $P$ values as exact values whenever suitable.

Х $\square$ For Bayesian analysis, information on the choice of priors and Markov chain Monte Carlo settings

Х $\square$ For hierarchical and complex designs, identification of the appropriate level for tests and full reporting of outcomes

$\bigotimes \square$ Estimates of effect sizes (e.g. Cohen's $d$, Pearson's $r$ ), indicating how they were calculated

Our web collection on statistics for biologists contains articles on many of the points above.

\section{Software and code}

Policy information about availability of computer code

Data collection The following software were used for data collection: For size exclusion chromatograhy, UNICORN software v5.31 (GE Life sciences); for ELISA readings, Magellan software V7.1 SP1 (Tecan Life Sciences); For neutralization and ADE assays, AID EliSpot software v8.0 (Autoimmun Diagnostika GMBH); for Flow cytometry, BD FACSDiva software v8.0.1 (BD Bioscience).

Data analysis

The following software were used for data analysis: For general analysis involving also statistical tests, Microsoft Excel 2016 software (Microsoft), SPSS software package (IBM) and GraphPad Prism software v8.0 (GraphPad Software Inc.); for analysis of Flow cytometry data, FlowJo software vX (FlowJo LLC).

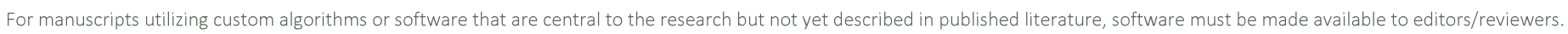
We strongly encourage code deposition in a community repository (e.g. GitHub). See the Nature Research guidelines for submitting code \& software for further information.

\section{Data}

Policy information about availability of data

All manuscripts must include a data availability statement. This statement should provide the following information, where applicable:

- Accession codes, unique identifiers, or web links for publicly available datasets

- A list of figures that have associated raw data

- A description of any restrictions on data availability

The authors declare that the data and materials supporting the findings discussed in this study are available within the publication, its supporting information or can be obtained upon request to the corresponding authors. 
Please select the one below that is the best fit for your research. If you are not sure, read the appropriate sections before making your selection.

$\bigotimes$ Life sciences $\quad \square$ Behavioural \& social sciences $\quad \square$ Ecological, evolutionary \& environmental sciences

For a reference copy of the document with all sections, see nature.com/documents/nr-reporting-summary-flat.pdf

\section{Life sciences study design}

All studies must disclose on these points even when the disclosure is negative.

Sample size Sample size was not statistically determined, samples sizes were chosen based on previous experience and taking into account the advice of the animal technicians and the $3 R$ reduction.

Data exclusions No data was excluded from the analysis.

Replication Due to limited sample availability, reproducibility was assess in at least 2 independent experiments done in duplicate. All attempts to reproduce experiments and data were successful.

Randomization No randomization was done. This is a immunization study in which all groups were composed of animals that share the same genetic background, sex distribution and age. Regardless of the group, animals were also treated equally throughout the study.

Blinding Investigators were not blinded, this had no effect on sample treatment or in the data reported. Regardless of the treatment received, all animal samples were analyzed at the same time and under the same conditions.

\section{Reporting for specific materials, systems and methods}

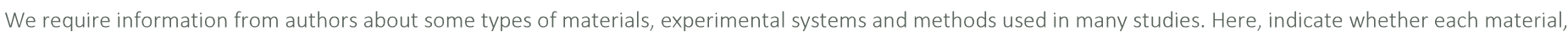

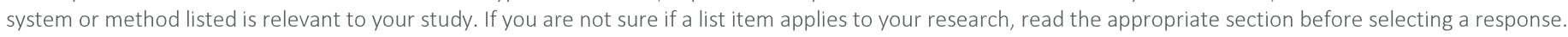

\begin{tabular}{l|l} 
Materials \& experimental sys \\
\hline$n / a$ & Involved in the study \\
$\square$ & $\bigotimes$ Antibodies \\
$\square$ & $\square$ Eukaryotic cell lines \\
$\square$ & $\square$ Palaeontology \\
$\square$ & $\square$ Human research participants \\
$\square$ & $\square$ Clinical data
\end{tabular}

\begin{tabular}{l|l} 
Methods \\
\hline n/a & Involved in the study \\
$\square$ & $\square$ ChIP-seq \\
$\square$ & $\bigotimes$ Flow cytometry \\
$\square$ & $\square$ MRI-based neuroimaging
\end{tabular}

\section{Antibodies}

Antibodies used

Horseradish peroxidase (HRP)-conjugated anti-mouse IgG (5220-0343, lot 10246668, 1:10,000) was purchased from SeraCare. FITC-conjugated anti-human Ig (F1085, lot 0095473, 1:200) and FITC-conjugated anti-mouse Ig (F0479, lot 20047943, 1:200) were purchased from Dako. Alkaline Phosphatase (ALP)-conjugated anti-mouse IgG+lgM (A9316, lot 061M6260, 1:2,000) and ALP-conjugated anti-human IgG (A9544, lot 026M4768, 1:5,000) were purchased from Sigma-Aldrich. Anti-Xpress antibody (R910-25, lot 1833004, 1:5,000) was purchased from Life Technologies. Anti-mouse Ifnar1 (Clone MAR1-5A3; I-1188, 10mg/mL) was purchased from Leinco. Mouse monoclonal anti-DENV E, 4G2, was a gift from AFRIMS. Monoclonal human anti-EDE1 (753-3C10, 752-2C8), anti-ED3 (ZA04-P4C5, ZA04-P5C10) and anti-FLE (758-P6B10, 759-B12) antibodies, as well as mouse antiSV5, and human anti-SV5 and anti-roTag (1F2) antibodies were produced as recombinant proteins and purified using Protein A/ G-conjugated agarose beads from PEI-transfected HEK293T cells. The DNA constructs required to produced anti-SV5 and antiroTag mAbs were kindly provided by Prof. Oscar R. Burrone (ICGEB, Trieste, Italy). For the panel of recombinant antibodies against DENV E, pairs of plasmids encoding heavy and light chain of human IgG1 were transfected in HEK293T and harvested. Antibody concentration was measured by ELISA and samples were used as culture supernatants.

Validation

All primary and secondary antibodies used in this study and listed above were previously validated by repeated down-titration to determine the optimal working concentration or dilution for each test. In each case, test runs and calibration tests were performed repeatedly and results were successfully reproduced. Although all validation was done and confirmed in-house, manufacturers information was taken into account, when available. 
Policy information about cell lines

Cell line source(s)

Vero and C6/36 cells were obtained from the Armed Forces Research Institute of Medical Sciences (AFRIMS), U397 cells were provided by Dr. Prida Malasit, HEK293T cells were provided by Prof. Xiaoning Xu

Authentication

Mycoplasma contamination

Commonly misidentified lines (See ICLAC register)
No authentication was done.

All cell lines and viral stocks were free from Mycoplasma

No commonly misidentified cell lines were used.

\section{Animals and other organisms}

Policy information about studies involving animals; ARRIVE guidelines recommended for reporting animal research

Laboratory animals

Wild animals

Field-collected samples

Ethics oversight
For assessment of immunogenicity, 6-8 weeks old, female inbred BALB/c ( $\mathrm{H}-2 \mathrm{~d})$ mice and 6-8 weeks old, female outbred CD1 mice were purchased from Envigo (UK). For viral challenge experiments, 6-8 weeks old, male and female C57BL/6J WT (000064) mice were purchased from Jackson Laboratories (USA).

\section{The study did not involve wild animals}

The study did not involve samples collected from the field

This study was carried out in accordance with the recommendations in the Guide for the Care and Use of Laboratory Animals of the National Institutes of Health and the UK Home Office Animals Act Project License. The protocols were approved by the Institutional Animal Care and Use Committee at the Washington University School of Medicine (Assurance number A3381-01), and by the University of Oxford Animal Care and Ethicl Review Committee (PPL 30/2414). Virus inoculations were performed under anaesthesia that was induced and maintained with ketamine hydrochloride and xylazine, and all efforts were made to minimize animal suffering.

Note that full information on the approval of the study protocol must also be provided in the manuscript.

\section{Flow Cytometry}

Plots

Confirm that:

\The axis labels state the marker and fluorochrome used (e.g. CD4-FITC).

$\bigotimes$ The axis scales are clearly visible. Include numbers along axes only for bottom left plot of group (a 'group' is an analysis of identical markers).

$\square$ All plots are contour plots with outliers or pseudocolor plots.

$\square$ A numerical value for number of cells or percentage (with statistics) is provided.

Methodology

Sample preparation

Instrument

Software

Cell population abundance

Gating strategy

Tick this box to confirm that a figure exemplifying the gating strategy is provided in the Supplementary Information.
Only yeast cells (Saccharomyces cerevisiae) were analysed by flow cytometry in this study. Cells were suspended in PBS-BSA 2\% during staining and inactivated with PBS-1\% formaldehyde before analysis.

BD LSRFortessa X20 flow cytometer (BD Biosciences)

For data collection, BD FACSDiva software v8.0.1 (BD Bioscience); for data analysis, FlowJo software vX (FlowJo LLC).

N/A

Bulk yeast cell population were selected in the preliminary FCS/SSC cell population plot and boundaries between "positive" and "negative" cells populations in the 488-530_30A channel (for FITC signal) where defined based on negative control cells (stained yeast cells without the primary antibody). 